\title{
RADAR AMBIGUITY FUNCTIONS, THE HEISENBERG GROUP, AND HOLOMORPHIC THETA SERIES
}

\author{
WALTER SCHEMPP ${ }^{1}$
}

\begin{abstract}
The concept of linear Schrödinger representation of the real Heisenberg nilpotent group and its various realizations is used to link the theory of radar ambiguity functions with nilpotent harmonic analysis. This group-representation theoretic approach allows us to analyze the radar ambiguity functions simultaneously in time and frequency. Moreover, it allows us to determine the group of all transformations that leave the radar ambiguity surfaces invariant and to specify all admissible envelope functions that belong to radar signals of the same finite energy. In particular, an investigation of the radial, i.e., $\mathrm{SO}(2, \mathbf{R})$-invariant radar ambiguity surfaces, gives rise to an identity for Laguerre-Weber functions of different orders, which implies on its part an identity for holomorphic theta series.
\end{abstract}

1. The basic group that stands at the crossroads of quantum mechanics and radar analysis is the real Heisenberg nilpotent group $\tilde{A}(\mathbf{R})$.

Indeed, the connected, simply connected two-step nilpotent Lie group $\tilde{A}(\mathbf{R})$ forms the group-theoretic embodiment of the Heisenberg canonical commutation relations of quantum mechanics (whence its name) which read at the Lie algebra level as follows:

$$
[X, Y]=T, \quad[X, T]=0, \quad[Y, T]=0 .
$$

The vectors $\{X, Y, T\}$ are generators of the real Heisenberg nilpotent Lie algebra $\mathfrak{n}$ with one-dimensional center $\mathbf{R} T$ and the exponential mapping exp: $\mathfrak{n} \rightarrow \tilde{A}(\mathbf{R})$ is a global diffeomorphism that carries Lebesgue measure on $\mathfrak{n}$ to Haar measure on $\tilde{A}(\mathbf{R})$.

On the other hand, let

$$
H(f, g ; x, y)=\int_{\mathbf{R}} f\left(t+\frac{1}{2} x\right) \bar{g}\left(t-\frac{1}{2} x\right) e^{2 \pi i y t} d t
$$

denote the cross-correlation function of two radar signal pulses which are reflected by a moving target. Here $f, g$ are the complex envelope functions of the signals involved. These envelopes are assumed to belong to the Schwartz space $S(\mathbf{R})$ of rapidly decreasing complex-valued smooth functions on the real line $\mathbf{R}$. Moreover, $x$ denotes the time delay, $y$ the Doppler frequency shift and $H(f, g ; \cdot, \cdot)$ the radar cross-ambiguity function. This function plays an important rôle in radar analysis and design (see Woodward [10]). In particular, $H(f, g ; \cdot, \cdot)$ allows us to formulate

Received by the editors June 9, 1983. Presented to the American Mathematical Society at the 797th Meeting, College Park, October 21, 1982, University of Maryland..

1980 Mathematics Subject Classification. Primary 22E25; Secondary 22E27, 22E30, 33A75, 43A80, 60G35, 81D05, 94A12.

${ }^{1}$ Supported by the Deutsche Forschungsgemeinschaft in Bonn. 
a geometric form of the radar uncertainty principle which parallels the uncertainty principle of quantum mechanics.

Let $U$ denote the linear Schrödinger representation of $\tilde{A}(\mathbf{R})$ acting on the complex Hilbert space $L^{2}(\mathbf{R})$; cf. Howe [3]. The Stone-von Neumann-Mackey theorem asserts that the representation $U$ of $\tilde{A}(\mathbf{R})$ is, up to unitary isomorphy, the only irreducible unitary linear representation of $\tilde{A}(\mathbf{R})$ with $t \rightsquigarrow e^{2 \pi i t}$ as central character. Moreover $S(\mathbf{R})$ admits the intrinsic definition as the complex vector subspace of smooth vectors for $U$. Let $\otimes$ denote the dyadic tensor product with respect to the prehilbert space structure of $S(\mathbf{R})$. Then we have the following grouprepresentation theoretic characterization of $H(f, g ; \cdot, \cdot)$.

THEOREM 1. Let $U$ denote the linear Schrödinger representation of the real Heisenberg nilpotent group $\tilde{A}(\mathbf{R})$ in its basic presentation (exponential coordinates). The radar cross-ambiguity function $H(f, g ; \cdot, \cdot)$ with respect to the envelope functions $f, g \in S(\mathbf{R})$ is the restriction of the coefficient function $\operatorname{tr}_{U}(f \otimes \bar{g})$ of $U$ to the polarized cross section to the center $\tilde{Z}$ in $\tilde{A}(\mathbf{R})$.

Let the differentiated version of the linear Schrödinger representation $U$ of $\tilde{A}(\mathbf{R})$ also be denoted by $U$. The following group-representation theoretic characterization of $H(f, g ; \cdot, \cdot)$ can be checked by means of the isotropic cross section to $\tilde{Z}$ in $\tilde{A}(\mathbf{R})$.

THEOREM 2. Consider the real Heisenberg nilpotent group $\tilde{A}(\mathbf{R})$ in its dual pairing presentation (canonical coordinates). Then the identity

$$
H(f, g ; p, q)=\operatorname{tr}_{\exp U}(\bar{g} \otimes f)(-p,-q)
$$

holds for all $(p, q) \in \mathbf{R} \times \mathbf{R}$.

2. In the terminology of electrical engineering,

$$
H(f ; x, y)=H(f, f ; x, y)=\int_{\mathbf{R}} f\left(t+\frac{1}{2} x\right) \bar{f}\left(t-\frac{1}{2} x\right) e^{2 \pi i y t} d t
$$

is called the radar auto-ambiguity function with respect to the signal envelope $f \in$ $S(\mathbf{R})$. The range of $H(f ; \cdot, \cdot)$ is called the radar auto-ambiguity surface (or energy distribution surface) generated by $f$. In the cross-ambiguity case the notation is similar. Since the linear Schrödinger representation $U$ of $\tilde{A}(\mathbf{R})$ is irreducible it follows from Theorem 1 by Choquet theory that the sum $H\left(f_{1} ; \cdot, \cdot\right)+H\left(f_{2} ; \cdot, \cdot\right)$ of any two radar auto-ambiguity functions with respect to signal envelope functions $f_{k} \neq 0$ in $S(\mathbf{R})$ that are not proportional is never a radar auto-ambiguity function. In particular, the mapping given by

$$
S(\mathbf{R}) \ni f \rightsquigarrow H(f ; \cdot, \cdot) \in S(\mathbf{R} \times \mathbf{R})
$$

is nonlinear.

Another important property of the linear Schrödinger representation $U$ of $\tilde{A}(\mathbf{R})$ is its square integrability $\bmod \tilde{Z}$, which on its part is equivalent to the geometric fact that the isomorphy class of $U$ corresponds under the natural bijection to a linear variety in $\mathfrak{n}^{*} / \operatorname{Coad}(\tilde{A}(\mathbf{R}))$ in Kirillov's orbit picture of the unitary dual of $\tilde{A}(\mathbf{R})$. In particular, the Schur orthogonality relations imply Moyal's identity

$$
\int_{\mathbf{R}} \int_{\mathbf{R}} H(f, g ; x, y) \bar{H}\left(f^{\prime}, g^{\prime} ; x, y\right) d x d y=\left\langle f \mid f^{\prime}\right\rangle\left\langle g^{\prime} \mid g\right\rangle
$$


where $f, f^{\prime}, g, g^{\prime}$ are elements of the Schwartz space $S(\mathbf{R})$.

It is well known that the fundamental group of the $\operatorname{symplectic} \operatorname{group} \operatorname{Sp}(1, \mathbf{R})=$ $\mathrm{SL}(2, \mathbf{R})$ is infinite cyclic so that there exists a unique connected Lie group $\mathrm{Mp}(1, \mathbf{R})$, called the metaplectic group, which forms a two-fold covering group of $\operatorname{Sp}(1, \mathbf{R})$; cf. Borel-Wallach [2]. Let $j: \tilde{\sigma} \rightsquigarrow \sigma$ denote the covering homomorphism so that

$$
\{0\} \rightarrow \mathbf{Z}_{2} \rightarrow \operatorname{Mp}(1, \mathbf{R}) \stackrel{j}{\longrightarrow} \operatorname{Sp}(1, \mathbf{R}) \rightarrow\{1\}
$$

forms a short exact sequence.

If $\tilde{\sigma} \rightsquigarrow T_{\tilde{\sigma}}$ denotes the metaplectic representation (or unitary oscillator representation in the language of physicists) of $\operatorname{Mp}(1, \mathbf{R})$ acting on $L^{2}(\mathbf{R})$, Theorem 1 yields the following result concerning the transformations that leave invariant the radar ambiguity surfaces generated by signals of the same finite energies.

THEOREM 3. Let $f, g$ denote complex signal envelope functions in $S(\mathbf{R})$ and let $\sigma \in \operatorname{Sp}(1, \mathbf{R})$ be given. Then we have

$$
H(f, g ; x, y)=H\left(T_{\tilde{\sigma}} f, T_{\tilde{\sigma}} g ; \sigma(x, y)\right)
$$

for all $(x, y) \in \mathbf{R} \times \mathbf{R}$. In particular, the radar auto-ambiguity function $H(f ; \cdot, \cdot)$ with respect to $f \neq 0$ is invariant under the cyclic subgroup of $\operatorname{Sp}(1, \mathbf{R})$ generated by $\{\sigma\}$ if and only if there exists a number $\zeta_{\tilde{\sigma}} \in \mathbf{T}$ such that $T_{\tilde{\sigma}} f=\zeta_{\tilde{\sigma}} f$ holds, i.e., if and only if $f \in S(\mathbf{R})$ is an eigenvector of the unitary operator $T_{\tilde{\sigma}}: L^{2}(\mathbf{R}) \rightarrow L^{2}(\mathbf{R})$.

Conversely, let $S$ denote a unitary automorphism of the complex prehilbert space $S(\mathbf{R})$. Suppose that for all pairs $(f, g)$ of functions in $S(\mathbf{R}) \times S(\mathbf{R})$ the radar crossambiguity functions $H(f, g ; \cdot, \cdot)$ and $H(S f, S g ; \cdot, \cdot)$ satisfy the following condition:

For any pair $(x, y) \in \mathbf{R} \times \mathbf{R}$ there exists a pair $\left(x^{\prime}, y^{\prime}\right) \in \mathbf{R} \times \mathbf{R}$ such that the identity

$$
H(f, g ; x, y)=H\left(S f, S g ; x^{\prime}, y^{\prime}\right)
$$

holds, i.e., the mappings $H(f, g ; \cdot, \cdot)$ and $H(S f, S g ; \cdot, \cdot)$ on $\mathbf{R} \times \mathbf{R}$ have the same range and therefore $(f, g)$ and $(S f, S g)$ generate the same radar cross-ambiguity surface.

Then there exists a unique mapping $\sigma \in \mathrm{Sp}(1, \mathbf{R})$ and a complex number $\eta \in \mathbf{T}$ such that

holds.

$$
\sigma(x, y)=\left(x^{\prime}, y^{\prime}\right), \quad S=\eta T_{\tilde{\sigma}}
$$

The same conclusion holds when the identity $(x)$ is merely supposed to hold on the diagonal of $S(\mathbf{R}) \times S(\mathbf{R})$, i.e., for the radar auto-ambiguity functions $H(f ; \cdot, \cdot)$ with respect to envelope functions $f \in S(\mathbf{R})$.

ProOF. Let the unitary group $\mathrm{U}\left(L^{2}(\mathbf{R})\right)$ of $L^{2}(\mathbf{R})$ be endowed with the strong operator topology and let the subgroup of intertwining operators

$$
G=\left\{S \in \mathbf{U}\left(L^{2}(\mathbf{R})\right) \mid S \circ U(v, s)=U\left(v^{\prime}, s\right) \circ S, v, v^{\prime} \in \mathbf{R} \oplus \mathbf{R}, s \in \mathbf{R}\right\}
$$

be equipped with the induced relative topology. Then $G$ has the structure of a Lie group and $v^{\prime}=h(S) v$, where $h(S): \mathbf{R} \oplus \mathbf{R} \rightarrow \mathbf{R} \oplus \mathbf{R}$ is a homeomorphism. The mapping $h: G \rightarrow \operatorname{Sp}(1, \mathbf{R})$ is a Lie group epimorphism and the irreducibility of $U$ implies via Schur's lemma that

$$
\{1\} \rightarrow \mathbf{T i d}_{L^{2}(\mathbf{R})} \rightarrow G \stackrel{h}{\rightarrow} \operatorname{Sp}(1, \mathbf{R}) \rightarrow\{1\}
$$


is a short exact sequence. Since $\operatorname{Mp}(1, \mathbf{R})$ is a subgroup of $G$, to wit the commutator group of $G$, and the Lie group homomorphism $h$ extends the covering projector $j$, and the unitary oscillator representation $T$ of $\operatorname{Mp}(1, \mathbf{R})$ coincides with the realization $\mathrm{Mp}(\mathbf{1}, \mathbf{R}) \hookrightarrow \mathbf{U}\left(L^{2}(\mathbf{R})\right)$, the Stone-von Neumann-Mackey theorem applied to $\tilde{A}(\mathbf{R})$ takes the form of the metaplectic formula

$$
T_{\tilde{\sigma}} \circ U(v, s) \circ T_{\tilde{\sigma}}^{-1}=U(\sigma(v), s) \quad(\sigma \in \operatorname{Sp}(1, \mathbf{R}))
$$

for all elements $(v, s) \in \tilde{A}(\mathbf{R})$. In view of Theorem 1 , the result follows from this formula by an application of the inversion theorem for the Fourier transform and a density argument.

The unitary automorphisms $T_{\tilde{\sigma}}$ of $S(\mathbf{R})$ can be computed explicitly in terms of polarizations of $\mathfrak{n}$ and suitable partial Fourier transforms. In particular, the preceding theorem allows us to determine the signal envelope functions that generate radar auto-ambiguity surfaces invariant under the cyclic group $\mathbf{Z}_{\mathbf{4}}$ of powers of a quarterturn about the origin of the time-frequency plane $\mathbf{R} \times \mathbf{R}$.

Let $\left(W_{m}\right)_{m \geq 0}$ denote the sequence of standardized Hermite-Weber functions (harmonic oscillator wave functions) and let the orthogonal direct sum

$$
L^{2}(\mathbf{R})=\bigoplus_{0 \leq k \leq 3} H_{k}
$$

be the Wiener decomposition of the complex Hilbert space $L^{2}(\mathbf{R})$. Thus the Hilbert sums

$$
\mathscr{H}_{k}=\widehat{\bigoplus} \bigoplus_{m \in \mathbf{N}} \mathbf{C} W_{4 m+k}, \quad k \in\{0,1,2,3\},
$$

denote the four eigenvector spaces of the Fourier cotransform $\overline{\mathcal{F}}_{\mathbf{R}}: L^{2}(\mathbf{R}) \rightarrow L^{2}(\mathbf{R})$ associated with its eigenvalues $i^{k}$ (of infinite multiplicities).

THEOREM 4. Let $f \neq 0$ be a given signal envelope function in $S(\mathbf{R})$. The condition $H(f ; x, y)=H(f ; y,-x)$ holds for all pairs $(x, y) \in \mathbf{R} \times \mathbf{R}$ if and only if $f \in \mathscr{H}_{k}$ for a (unique) number $k \in\{0,1,2,3\}$.

ProOF. The Fourier cotransform $\overline{\mathcal{F}}_{\mathbf{R}} \in \mathbf{U}\left(L^{2}(\mathbf{R})\right)$ belongs to $G$ and its image under the Lie group epimorphism $h: G \rightarrow \operatorname{Sp}(1, \mathbf{R})$ is the Cauchy-Riemann matrix

$$
J=\left(\begin{array}{cc}
0 & +1 \\
-1 & 0
\end{array}\right) \text {. }
$$

Thus $f \in S(\mathbf{R})$ has to be an eigenfunction of $\overline{\mathcal{F}}_{\mathbf{R}}$ by Theorem 3 supra.

Obviously the Hermitian central symmetry $H(f ; x, y)=\bar{H}(f ;-x,-y)$ holds for any $f \in S(\mathbf{R})$ and all pairs $(x, y) \in \mathbf{R} \times \mathbf{R}$. In particular, the radar auto-ambiguity functions invariant under the cyclic group $\mathbf{Z}_{4}$ of quaterturns about the origin of $\mathbf{R} \times \mathbf{R}$ are real valued.

Identify the Lie algebra of $\operatorname{Mp}(1, \mathbf{R})$ with $\mathfrak{s} p(1, \mathbf{R})=\mathfrak{s l}(2, \mathbf{R})$ and denote by Exp the exponential mapping of $s p(1, \mathbf{R})$ into $M p(1, \mathbf{R})$. Consider the CauchyRiemann matrix $J$ as an element of $s p(1, \mathbf{R})$. Then $\operatorname{Exp}(\mathbf{R} J)$ is a Cartan subgroup of $\operatorname{Mp}(1, \mathbf{R})$ and $h(\operatorname{Exp}(\mathbf{R} J))$ is a Cartan subgroup of $\operatorname{Sp}(1, \mathbf{R})$. Moreover, let $T^{-}=-\frac{1}{2}(X-i Y), T^{+}=-\frac{1}{2}(X+i Y)$. Then $a^{-}=U\left(T^{-}\right), a^{+}=U\left(T^{+}\right)$are the boson annihilation and creation operators, respectively, and

$$
a^{-}\left(W_{0}\right)=0, \quad W_{m}=\left(\pi^{m} m !\right)^{-1 / 2}\left(a^{+}\right)^{m}\left(W_{0}\right), \quad m \in \mathbf{N}
$$


holds. The irreducibility of the linear Schrödinger representation $U$ of $\tilde{A}(\mathbf{R})$ implies the well-known fact that the family $\left(W_{m}\right)_{m \geq 0}$ of standardized Hermite-Weber functions forms a Hilbert basis of $L^{2}(\mathbf{R})$. It is easy to check that the identities

$$
\begin{gathered}
T_{\operatorname{Exp}(r J)} a^{-} T_{\operatorname{Exp}(-r J)}=e^{-2 \pi i r} a^{-}, \quad T_{\operatorname{Exp}(r J)} a^{+} T_{\operatorname{Exp}(-r J)}=e^{+2 \pi i r} a^{+}, \\
T_{\operatorname{Exp}(r J)} W_{0}=e^{\pi i r} W_{0},
\end{gathered}
$$

hold on $S(\mathbf{R})$ where $r \in \mathbf{R}$. Thus the elements of the Hilbert basis $\left(W_{m}\right)_{m \geq 0}$ of $L^{2}(\mathbf{R})$ are simultaneously eigenfunctions of the subgroup $T_{\operatorname{Exp}(\mathbf{R} J)}$ of $\mathbf{U}\left(L^{2}(\mathbf{R})\right)$. Conversely, any Hilbert basis of $L^{2}(\mathbf{R})$, the elements of which belong to $S(\mathbf{R})$ and are simultaneously eigenfunctions of the group $T_{\operatorname{Exp}(\mathbf{R} J)}$, forms a total family of eigenfunctions of the Hermite linear differential operator of second order

$$
2\left(a^{-} a^{+}+a^{+} a^{-}\right)=2 i(d / d r)_{r=0} T_{\operatorname{Exp}(r J)}
$$

with domain $S(\mathbf{R})$. This operator is essentially selfadjoint in $L^{2}(\mathbf{R})$, its closure admits a pure point spectrum given by $\{-2 \pi(2 m+1) \mid m \in \mathbf{N}\}$ and all of its eigenvalues are simple. Consequently the following result is proven.

THEOREM 5. Let the signal envelope function $f \in S(\mathbf{R})$ with $L^{2}$-norm $\|f\|=1$ be given. The radar auto-ambiguity function $H(f ; \cdot, \cdot)$ is radially symmetric, i.e., $\mathrm{SO}(2, \mathbf{R})$-invariant on the time-frequency plane $\mathbf{R} \times \mathbf{R}$ if and only if $f=\varsigma W_{m}$ holds for certain numbers $m \in \mathbf{N}$ and $\zeta \in \mathbf{T}$. Thus aside from a phase factor $f$ is one of the harmonic oscillator wave functions $W_{m}$.

REMARK. By an application of the theorems of Bochner and Hadamard the standardized Gaussian $W_{0} \in S(\mathbf{R})$ can be characterized in the following way. Let $f$ be as in Theorem 5 supra. The radar auto-ambiguity function $H(f ; \cdot, \cdot)$ is $\mathrm{SO}(2, \mathbf{R})$ invariant and of positive type on the time-frequency plane $\mathbf{R} \times \mathbf{R}$ if and only if $f=\varsigma W_{0}$.

In view of Moyal's identity supra the family $\left(H\left(W_{m}, W_{n} ; \cdot, \cdot\right)\right)_{m \geq 0, n \geq 0}$ forms a Hilbert basis of $L^{2}(\mathbf{R} \times \mathbf{R})$. In order to determine explicitly the elements of this Hilbert basis let $\left(L_{n}^{(\alpha)}\right)_{n \geq 1}$ denote the sequence of Laguerre-Weber functions of order $\alpha>-1$. An application of Theorem 2 supra yields (cf. Wilcox [9]):

THEOREM 6. For all $(p, q) \in \mathbf{R} \times \mathbf{R}$ the identities

$$
\begin{array}{ll}
H\left(W_{m}, W_{n} ; p, q\right)=\sqrt{\frac{n !}{m !}}(\sqrt{\pi}(p+i q))^{m-n} L_{n}^{(m-n)}\left(\pi\left(p^{2}+q^{2}\right)\right) & (m \geq n), \\
H\left(W_{m}, W_{n} ; p, q\right)=\sqrt{\frac{m !}{n !}}(\sqrt{\pi}(-p+i q))^{n-m} L_{m}^{(n-m)}\left(\pi\left(p^{2}+q^{2}\right)\right) & (n \geq m)
\end{array}
$$

hold. In particular, we have

$$
H\left(W_{m} ; p, q\right)=L_{m}^{(0)}\left(\pi\left(p^{2}+q^{2}\right)\right)
$$

for all $m \geq 0$.

PROOF. Let $\tilde{\delta}$ denote the right regular representation of $\tilde{A}(\mathbf{R})$ on $L^{2}(A(\mathbf{R}) / \tilde{Z})$ transferred to $L^{2}(\log \mathbf{R} \oplus \log \mathbf{R})$ by the exponential mapping. Then we get by 
Theorem 2 supra

$$
\begin{aligned}
H\left(W_{m}, W_{n} ; p, q\right) & =\operatorname{tr}_{\exp U}\left(W_{n} \otimes W_{m}\right)(-p,-q)=\left\langle W_{n} \mid e^{U(p X+q Y)}\left(W_{m}\right)\right\rangle \\
& =\frac{1}{\sqrt{\pi^{m} m !}}\left\langle W_{n} \mid e^{U(p X+q Y)}\left(a^{+}\right)^{m}\left(W_{0}\right)\right\rangle \quad(m, n \in \mathbf{N}) \\
& =\frac{1}{\sqrt{\pi^{m} m !}} \tilde{\delta}\left(T^{+}\right)^{m}\left\langle W_{n} \mid e^{U(p X+q Y)}\left(W_{0}\right)\right\rangle \\
& =\frac{(-1)^{m}}{2^{m} \sqrt{\pi^{m-n} m ! n !}} e^{(\pi / 2)\left(p^{2}+q^{2}\right)}\left(\frac{\partial}{\partial p}+i \frac{\partial}{\partial q}\right)^{m}(-p+i q)^{n} e^{-\pi\left(p^{2}+q^{2}\right)}
\end{aligned}
$$

for all $(p, q) \in \mathbf{R} \times \mathbf{R}$. Supposing $m \geq n$, we see that

$$
\begin{aligned}
& H\left(W_{m}, W_{n} ; p, q\right) \\
& \quad=\sqrt{\frac{n !}{\pi^{m-n} m !}} e^{-(\pi / 2)\left(p^{2}+q^{2}\right)} \sum_{0 \leq j \leq n}\left(\begin{array}{c}
m \\
j
\end{array}\right) \frac{1}{(n-j) !}(\pi(p+i q))^{m-j}(-p+i q)^{n-j} \\
& =\sqrt{\frac{n !}{m !}} e^{-(\pi / 2)\left(p^{2}+q^{2}\right)}(\sqrt{\pi}(p+i q))^{m-n} \sum_{0 \leq j \leq n}\left(\begin{array}{c}
m \\
j
\end{array}\right) \frac{1}{(n-j) !}\left(-\pi\left(p^{2}+q^{2}\right)\right)^{n-j}
\end{aligned}
$$

holds. Inserting the expression

$$
L_{n}^{(m-n)}(x)=e^{-x / 2} \sum_{0 \leq j \leq n}\left(\begin{array}{c}
m \\
j
\end{array}\right) \frac{(-x)^{n-j}}{(n-j) !} \quad(m \geq n)
$$

the result follows.

3. Let $D$ denote the image of the cubic integer lattice $\mathbf{Z}^{3}$ under the natural embedding map into $\tilde{A}(\mathbf{R})$. Then $D$ is a discrete cocompact subgroup of $\tilde{A}(\mathbf{R})$ and the homogeneous space $D \backslash \tilde{A}(\mathbf{R})$ of right cosets modulo $D$ forms a two-step compact nilmanifold, called the Heisenberg nilmanifold. It carries a unique probability measure invariant under right translations. Decompose the complex Hilbert space $L^{2}(D \backslash \tilde{A}(\mathbf{R}))$ into the Hilbert sum

$$
L^{2}(D \backslash \tilde{A}(\mathbf{R}))=\bigoplus_{n \in \mathbf{Z}} M_{n}
$$

of primary summands with respect to the right quasi-regular representation $\delta$ of $\tilde{A}(\mathbf{R})$ on $L^{2}(D \backslash \tilde{A}(\mathbf{R}))$. The subduced representation $\delta_{1}$ of $\tilde{A}(\mathbf{R})$ acting on $M_{1}$ is irreducible and therefore, by the Stone-von Neumann-Mackey theorem, unitarily isomorphic to the linear Schrödinger representation $U$ of $\tilde{A}(\mathbf{R})$. Indeed, the WeilBrezin map (already known to C. F. Gauss)

$$
w_{1}: S(\mathbf{R}) \rightarrow M_{1} \cap C^{\infty}(D \backslash \tilde{A}(\mathbf{R}))
$$

extends to a unitary isomorphism $L^{2}(\mathbf{R}) \rightarrow M_{1}$ of $U$ onto $\delta_{1}$.

THEOREM 7. The radar cross-ambiguity function $H(f, g ; \cdot, \cdot)$ with respect to the signal envelope functions $f, g \in S(\mathbf{R})$ is the restriction of the trace evaluation $\operatorname{tr}_{\delta_{1}}\left(w_{1}(f) \otimes \overline{w_{1}(g)}\right)$ to the polarized cross section to $\tilde{Z}$ in $\tilde{A}(\mathbf{R})$. In particular, we have

$$
\left(w_{1}(f) \cdot \overline{w_{1}(g)}\right)(x, y, 0)=\sum_{(\mu, \nu) \in \mathbf{Z} \times \mathbf{Z}} H(f, g ; \mu, \nu) e^{2 \pi i(-\nu x+\mu y)}
$$


for $(x, y) \in \mathbf{R} \times \mathbf{R}$ with respect to the topology of uniform $C^{\infty}$-convergence.

The Parseval-Plancherel theorem combined with Theorems 7 and 6 gives

THEOREM 8. For all pairs $(m, n) \in \mathbf{N} \times \mathbf{N}$ the identities

$$
\sum_{(\mu, \nu) \in \mathbf{Z} \times \mathbf{Z}} H\left(W_{m} ; \mu, \nu\right) \cdot H\left(W_{n} ; \mu, \nu\right)=\sum_{(\mu, \nu) \in \mathbf{Z} \times \mathbf{Z}}\left|H\left(W_{m}, W_{n} ; \mu, \nu\right)\right|^{2}
$$

hold ("Poisson-Plancherel identities" for radially symmetric radar auto-ambiguity functions). In particular, we have

$$
\begin{aligned}
\sum_{(\mu, \nu) \in \mathbf{Z} \times \mathbf{Z}} & L_{m}\left(\pi\left(\mu^{2}+\nu^{2}\right)\right) L_{n}\left(\pi\left(\mu^{2}+\nu^{2}\right)\right) \\
= & \frac{n !}{m !} \pi^{m-n} \sum_{(\mu, \nu) \in \mathbf{Z} \times \mathbf{Z}}\left(\mu^{2}+\nu^{2}\right)^{m-n}\left(L_{n}^{(m-n)}\left(\pi\left(\mu^{2}+\nu^{2}\right)\right)\right)^{2}
\end{aligned}
$$

when $m \geq n$ and $L_{m}=L_{m}^{(0)}$.

In the preceding identity the simplest case occurs when $m=1, n=0$. Then we get the following relation between holomorphic theta series,

$$
\frac{1}{4 \pi} \sum_{\mu \in \mathbf{Z}} e^{-\pi \mu^{2}}=\sum_{\mu \in \mathbf{Z}} \mu^{2} e^{-\pi \mu^{2}}
$$

which is well known from the theory of classical Jacobi theta functions. These functions are deeply connected with some versions of theta functions, the so-called nil-theta functions that are living in a natural way on the Heisenberg compact nilmanifold $D \backslash \tilde{A}(\mathbf{R})$; cf. Auslander [1] and Mumford [4].

Further details will appear in [6-8]. For applications of nilpotent harmonic analysis to digital signal processing, see [5].

ACKNOWLEDGements. It is a pleasure to thank Professors Luigi Gatteschi, Bruno Gabutti, and Dr. Silvano Straneo for the hospitality of the Istituto di Calcoli Numerici dell'Università di Torino where part of this work was done. Moreover, the author is grateful to the Consiglio Nazionale delle Ricerche of Italy for financial support.

\section{REFERENCES}

1. L. Auslander, Lecture notes on nil-theta functions, CBMS Regional Conf. Ser. in Math., no. 34, Amer. Math. Soc., Providence, R.I., 1977.

2. A. Borel and N. Wallach, Continuous cohomology, discrete subgroups, and representations of reductive groups, Ann. of Math. Studies, no. 94, Princeton Univ. Press, Princeton, N.J., 1980.

3. R. Howe, Quantum mechanics and partial differential equations, J. Funct. Anal. 38 (1980), 188254.

4. D. Mumford, Tata lectures on theta. I, Progress in Math., Vol. 28, Birkhäuser, Boston, Mass., 1983.

5. W. Schempp, Gruppentheoretische Aspekte der Signalübertragung und der kardinalen Interpolationssplines. I, Math. Methods Appl. Sci. 5 (1983), 195-215.

6. __ Radar ambiguity functions, nilpotent harmonic analysis, and holomorphic theta series, Special Functions. Group Theoretic Aspects and Applications (R. A. Askey, T. H. Koornwinder, W. Schempp, eds.), Reidel, Dordrecht, 1984.

7. __ Harmonic analysis on the Heisenberg group with applications, Pitman, Boston, Mass. 
8. _ The complex Laplace-Beltrami operator, nilpotent harmonic analysis, and holomorphic theta series (to appear).

9. C. H. Wilcox, The synthesis problem for radar ambiguity functions, MRC Tech. Summary Report \#157, 1960.

10. P. M. Woodward, Probability and information theory with applications to radar, 2nd ed., Pergamon Press, New York, 1964.

Lehrstuhl fuer Mathematik I Der Universitaet Siegen, D-5900 Siegen, FeDERAL REPUBLIC OF GERMANY 